\title{
INCREASING ACCURACY OF THE MOBILE ROBOT POSITIONING SYSTEM BY USING ARTAGS
}

\author{
R. Kazala* ${ }^{*}$ P. Straczynski ${ }^{* *}$
}

\begin{abstract}
The article presents the results of research related to the development of a multi-sensor navigation system for a mobile robot. Common methods for determining the robot's position are discussed. Next, a navigation system for a mobile robot was proposed that uses a vision system and AR tags. A processing algorithm that allows to increase positioning accuracy in a robot control system by detecting markers is presented. An implemented AR tag recognition system is shown. Conclusions on the usefulness of the presented solution are presented.
\end{abstract}

Keywords: navigation system, localization, mobile robots, vision system

\section{Introduction}

In recent years, the rapid development of mobile robots has been visible. Mobile robots are used in the exploration of planets and dangerous places, by uniformed services for the transfer of explosives, in warehouse transport systems and as cleaning devices. A very large increase in applications can be observed in the group of unmanned flying vehicles (drones), which constitute a special group of mobile robots. Vehicles of this type can be used, among others, in agriculture, (checking the condition of crops or spraying them) or geodesy (making orthophotomaps). In the future, unmanned vehicles can have a big role in merchandise shipments, for example Amazon is working on this type of service as part of the Prime Air project. Due to the aging of people in the future, robots will be widely used as domestic help. The great popularity of mobile robots and dynamic growth of research on autonomous systems carries the need for the development of inexpensive navigation systems. The paper presents the results of research related to the development of a multi-sensor navigation system for a mobile robot.

\section{An overview of the methods used to determine the location and orientation of robots}

Currently, many methods are used in robotics to determine the position. We can divide them into direct and incremental methods. The most popular method of measuring the immediate position of the object is the use of a satellite navigation system, such as GPS, Galileo or GLONASS. Similarly direct measurement is ensured by radio navigation (using radio signals in the positioning system) or systems using distance measurement with special reference objects (Hallman, 2007). Despite the many advantages of systems based on direct measurement of positions, these methods also have many disadvantages. The use of civilian GPS receivers is associated with low accuracy. There is also a problem with using them inside buildings. Relatively high accuracy provides positioning based on a markers or reference radio transmitters. The last group of navigational systems that is able to directly determine the position of the object (robot, drone) is a navigation using reflective distance sensors or lidars which determine the position with respect to other objects. With this method, a tracking object can only move in a known environment. A separate group of location methods are incremental methods. The location at a given moment is determined on the basis of knowledge about the movement made and the position of the object at the initial moment.

Robert Kazala, PhD.: Department of Industrial Electrical Engineering and Automatics, Kielce University of Technology, Aleja Tysiaclecia Panstwa Polskiego 7; 25-314 Kielce; PL, rkazala@tu.kielce.pl

** Pawel Straczynski, MSc.: Department of Industrial Electrical Engineering and Automatics, Kielce University of Technology, Aleja Tysiaclecia Panstwa Polskiego 7; 25-314 Kielce; PL, pstraczynski@tu.kielce.pl 
In practice, it is often impossible to effectively use navigation based on the INS system as is the case with sea or air navigation. The large price of good quality inertial sensors precludes their use in many applications. Measurements from low-cost sensors made in MEMS technology contain high noise. This causes an increasing accumulation of errors in measuring the position as a function of time. This is related to the need to perform a double integration operation. However, this type of systems can be successfully used as a support system for satellite navigation, eg in the moments of signal loss. The multitude of possible solutions means that the selection of the appropriate location method should be chosen individually in a given application, often including the fusion of sensory data.

\section{The location system which uses the GPS receiver and elements of the INS system.}

The structure of a mobile robot positioning system using a GPS receiver and inertial navigation components is shown in Figure 1.

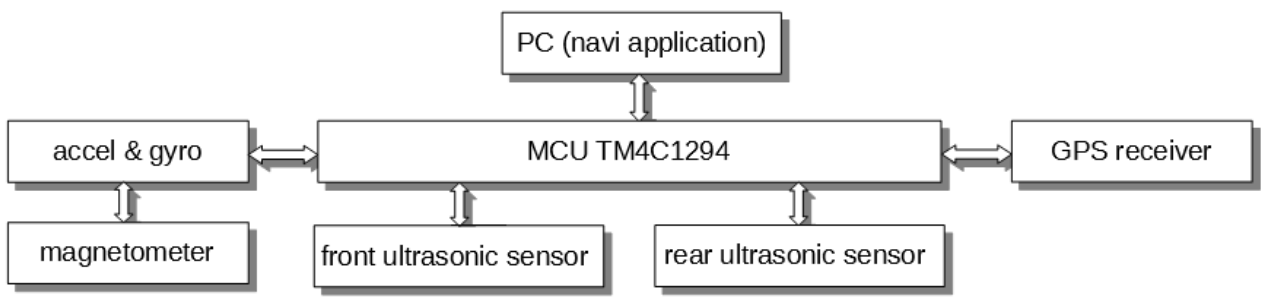

Fig. 1: Mobile robot navigation system - block diagram

As shown in the figure, data acquisition from sensors is handled by a 32-bit microcontroller with an ARM Cortex-M4F core. The data is pre-processed and transmitted to a PC computer using Ethernet. The current robot position (determined on the basis of the GPS receiver) is displayed on the map. In addition, the user can define a destination point. The task of the application is to designate special navigational coordinates. To determine the distance between the current location and the destination point, the Haversine formula was used, which can be described using the following formulas

$$
\begin{gathered}
a=\sin ^{2} \frac{\Delta \Theta}{2}+\cos \frac{\Theta_{1}}{2}+\cos \frac{\Theta_{2}}{2}+\sin ^{2} \frac{\phi}{2} \\
l=2 \cdot \operatorname{atan} 2(\sqrt{a}, \sqrt{1-a}) \cdot R
\end{gathered}
$$

where: $\Delta$ - the difference between the lengths, $\Theta_{1}$ - the geographical length of the robot's position, $\Theta_{2}$ longitude the destination point, $\Delta \phi$ - the difference between the geographical latitudes of both points, $\mathrm{R}$ average length of the Earth's radius, 1 - distance between points.

Based on the data from the magnetometer and the above data, it is possible to calculate the angle by which the robot has to rotate in order to reach the destination point (Straczynski, 2016).

$$
\alpha=\operatorname{atan} 2\left(\sin \Delta \phi \cdot \cos \Theta_{1}, \cos \Theta_{2}-\sin \Theta_{1} \cdot \cos \Theta_{2} \cdot \cos \Delta \phi\right)
$$

\section{Extension of the positioning system with the detection of ARTag markers}

Based on field tests performed, the positioning system presented in the previous section does not work well. In the case of large distances between the target and the current position, the robot is guided correctly towards the target but does not reach it. In situations where the distance between the target and the current position of the robot is small (several meters) the accuracy of the GPS receiver excludes effective positioning. Because the robot's sensory system contains low-cost inertial sensors, which eliminates the possibility of increasing the accuracy of positioning based on the acceleration measurement, it was decided to extend the navigation system with a vision system based on the detection of ARTag markers (Fiala, 2005). The 
positioning application based on tags was written in Python using the OpenCV library. The basic tasks of the program are:

- pre-processing of the image,

- detection of known ARTag markers,

- determination of distances to markers,

- determining the position of the robot,

- sending data to the control application.

During testing relationships between changes in the threshold at the stage of image binarization and the effectiveness of markers detection have been observed. The first approach to solving the problem was based on the adaptive thresholding algorithm (Bradley et al., 2007). In our research, the use of similar algorithms did not give satisfactory results. Therefore, it was decided to apply global thresholding with a variable level.

$$
\mathbf{g}(\mathbf{x}, \mathbf{y})= \begin{cases}1 & \text { if } f(x, y) \geq \mathbf{T} \\ 0 & \text { otherwise }\end{cases}
$$

where $\mathbf{g}(\mathbf{x}, \mathbf{y})$ is a vector of arrays and $\mathbf{T}$ is a vector of coefficients. This allowed us to correctly detect more markers.

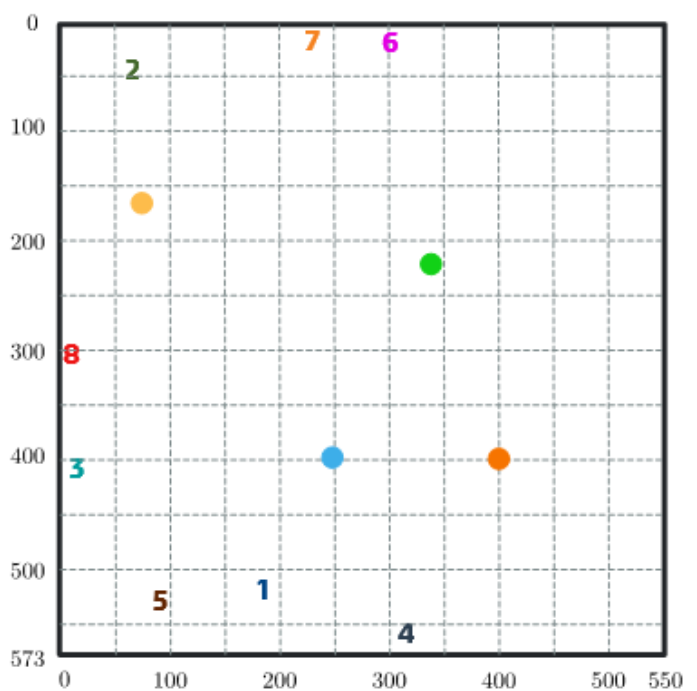

Markers

Q $1(178,511)$

W. $2(68,33)$

Q $3(15,403)$

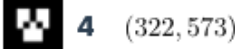

W 5 (48,477)

W $6(299,0)$

4. $7 \quad(232,0)$

니 $8(0,300)$
Robot position

$(68,158)$

$(340,219)$

$(400,400)$

$(249,400)$

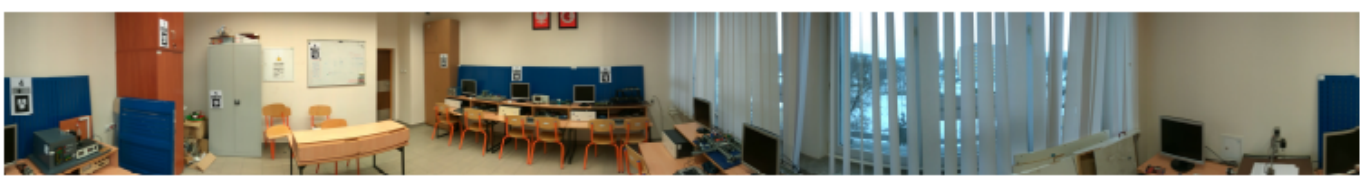

Fig. 2: Test area map with marked measurement points and markers

At the stage of initial processing, the color image is converted to a scale of gray. After the series of tests, we decided to abandon the image filtering operation, which usually aims to reduce noise. algorithm of data processing algorithm of data processing algorithm of data processing After thresholding, the image undergoes the typical operation in this case: detection of axes (quadrangles), perspective transformation of found objects and comparison with patterns. Based on the size of the detected marker, the distance between the camera and the marker is estimated. The angle between the robot's direction and the marker is estimated based on the area of the image in which the marker was detected. The results of operations in the individual processing phases are summed up. The test area is shown in the Figure 2.

On the basis of the analysis presented in the work (Cohen et al., 1992), it was decided to use the method of intersecting circles. In real conditions, due to the error of distance and angle measurements, the circles do 
not intersect at one point. The task of the data processing system is to select from the set of six points (crosssection) the three most-collected. In the presented system, it was proposed to use the k-nearest neighbors algorithm for this purpose. Implementation of this method is included in scikit-learn, the machine learning library for Python. Next, the three coordinate points are averaged to estimate the current position of the robot. The processing algorithm is shown in the Figure 3. Position data is sent to the control application using the MQTT protocol (Dudek et al., 2016). The result of the algorithm test is shown in the Figure 4.

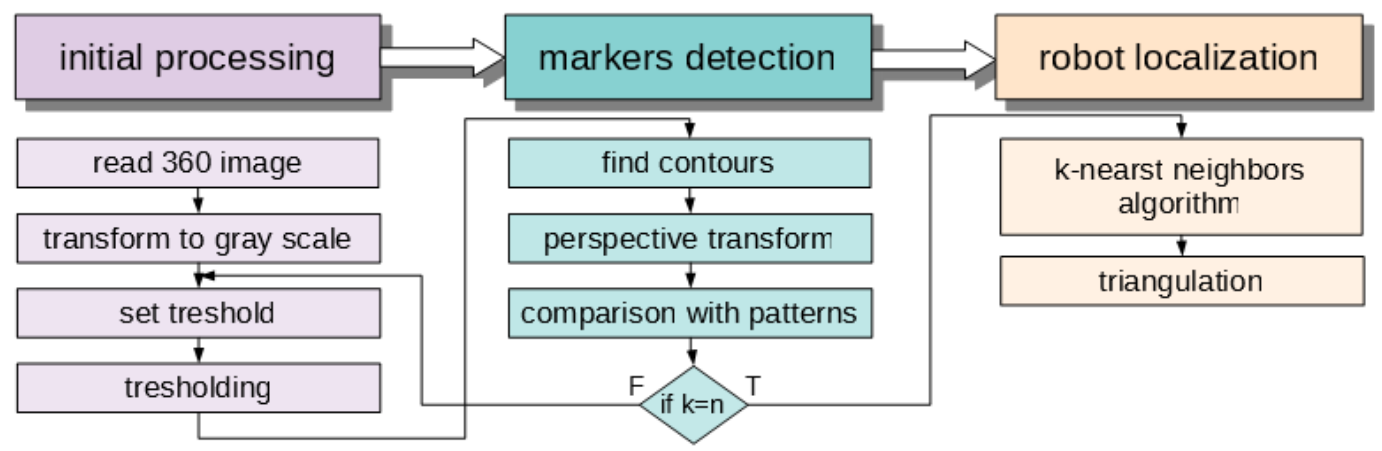

Fig. 3: Signal processing algorithm

a)

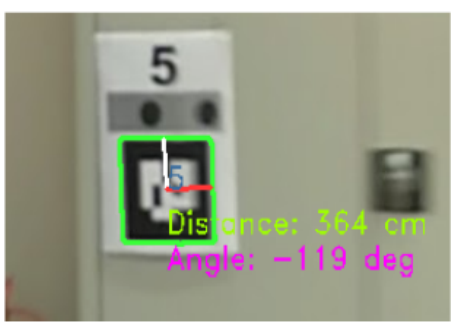

b)

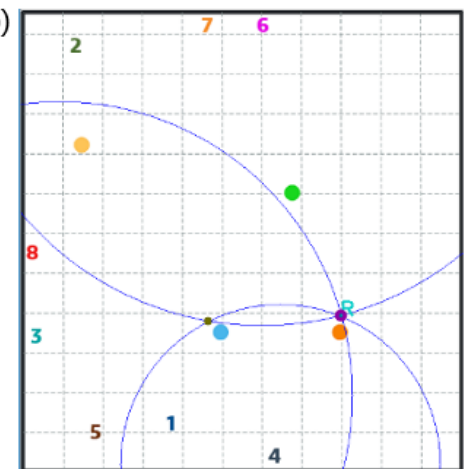

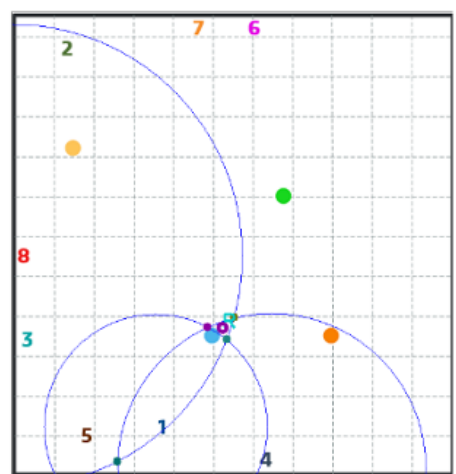

Fig. 4: Result of processing: a) ARTag detect, b) robot localization

\section{Conclusions}

The research results presented in the work confirm that the use of AR markers and vision system improves positioning accuracy in the mobile robot control system. This is particularly important in applications where no GPS signal is available, and inertial sensors do not provide sufficient accuracy. The presented image processing algorithm increases the probability of detecting many markers, and thus allows to increase the positioning accuracy. In the future, it is planned to develop the system through the use of complex data fusion algorithms.

\section{References}

Bradley, D. and Roth, G. (2007) Adaptive Thresholding Using Integral Image. Journal of Graphics Tools. 12(2) 13-21. Cohen, C. and Koss, F. (1992), A comprehensive study of three-object triangulation, In: Proc. SPIE 1831, Mobile Robots VII, (4 May 1993); doi: 10.1117/12.143782

Dudek, D., Kazala, R., and Straczynski, P. (2017) Jitter analysis of MQTT protocol frames in mobile robot control system. In: Engineering Mechanics 2017, Brno University of Technology, Brno, 286-289.

Dudek, D., Kazala, R., and Straczynski, P. (2016) Mobilny robot manipulacyjny wykorzystujacy technologie Internetu Rzeczy w systemie sterowania i monitorowania. Pomiary Automatyka Robotyka, 20(4) 37-45.

Fiala, M. (2005) ARTag, a fiducial marker system using digital techniques. In: 2005 IEEE Computer Society Conference on Computer Vision and Pattern Recognition, IEEE, pp. 590-596.

Hallman, I. (2007) Lokalizacja robota mobilnego wzgledem automatycznie wybieranych obiektow. Dissertation. Instytut Podstawowych Problemów Techniki, Warszawa.

Straczynski, P. (2016) System nawigacji i sterowania robotem mobilnym wykorzystujacy mikrokontroler Arm. Diploma thesis. Kielce University of Technology, Kielce. 